\title{
A Tiered Hydrologic and Hydraulic Modeling System to Satisfy Multiple Objectives in Complex Sewershed Applications
}

\author{
James T. Smullen, Edward H. Burgess and Robert E. Dickinson
}

\begin{abstract}
A hydrologic and hydraulic modeling system was developed in two stages, or tiers, for use in large-scale, comprehensive planning studies for watersheds and sewersheds in complex combined and separate sewered systems. The initial suite of models is based upon a detailed representation of important hydraulic elements, for instance combined sewer overflow and sanitary sewer overflow regulator and interceptor hydraulics, and a simplified representation of watershed and sewershed hydrology. The second tier of models, relies upon a more complete representation of hydraulic elements and a more detailed representation of basin hydrology. The principal purpose for taking this incremental or evolutionary approach to model development is to satisfy changing needs of large-scale planning projects during the entire project period, not just at the end. This approach provides opportunities to make model-based evaluation capabilities available "just-in-time" to address project needs for interim outputs and to answer questions as they arise throughout the life of the project.
\end{abstract}

\subsection{The Need for a Tiered Modeling Approach}

The demand for large-scale watershed planning studies in North America has been increasing steadily over the past ten years. In large part, the demand is driven by major government programs regulating stormwater discharges,

Smullen, J., E. Burgess and R.E. Dickinson. 2002. "A Tiered Hydrologic and Hydraulic Modeling System to Satisfy Multiple Objectives in Complex Sewershed Applications." Journal of Water Management Modeling R208-01. doi: 10.14796/JWMM.R208-01.

(c) CHI 2002 www.chijournal.org ISSN: 2292-6062 (Formerly in Best modeling practices for Urban Water Systems. ISBN: 0-9683681-6-6) 
combined sewer overflows, sanitary sewer overflows and, most recently, programs limiting the maximum allowable pollutant discharges allowed to enter waterways from all sources. The implementation of these regulatory programs most often results in a local or regional public agency embarking upon large, multi-year studies requiring comprehensive inventory and characterization of watershed and sewershed hydraulic, hydrologic and water polluting elements and processes. The agencies implementing these programs typically are required to provide major milestone reports at varying times during the project, and the analytical approaches chosen must be available in time and at an appropriate level of complexity to support the development of those reports. Traditional modeling approaches that involve development at very high levels of hydrologic and hydraulic detail everywhere in a system seldom are capable of supporting assessments prior to the latter stages of the project. What is needed is a different modeling approach, one that allows for system-wide assessments to be supported early enough in the project to influence results and conclusions needed to satisfy interim regulatory reporting requirements.

\subsection{Tiered Modeling Approach}

Computer-based models of sewershed hydrology and trunk or interceptor hydraulics typically are developed for comprehensive studies of complex sewer systems. The development of the computer models of entire major sewer systems is a large undertaking requiring the application of significant resources, including experienced computer modelers, computer systems and technical support staff, over an extended period of time. Rather than develop a large, detailed computer model by building sequentially a detailed representation of each sub-system, it is preferable to build the model as an evolutionary process, employing the appropriate tools at various stages in the process to meet different objectives as they evolve. In this manner, the model takes on different forms during the process, with some elements of the system initially modeled in greater detail than others, depending upon the specific level of detail required to support any given phase of the sewer overflow planning process. This concept is expressed graphically in Figure 1.1.

The multi-phased hydraulic and hydrologic model development process focuses the greatest detail initially on the sewer system, using the USEPA Storm Water Management Model (SWMM) Extended Transport (EXTRAN) module, in a first phase, or Tier 1 modeling effort. The EXTRAN module of SWMM is the most widely used and accepted model for interceptor, combined sewer overflow (CSO) and sanitary sewer overflow (SSO) modeling (Roesner et al., 1988). It accurately simulates complex hydraulic conditions that occur in 

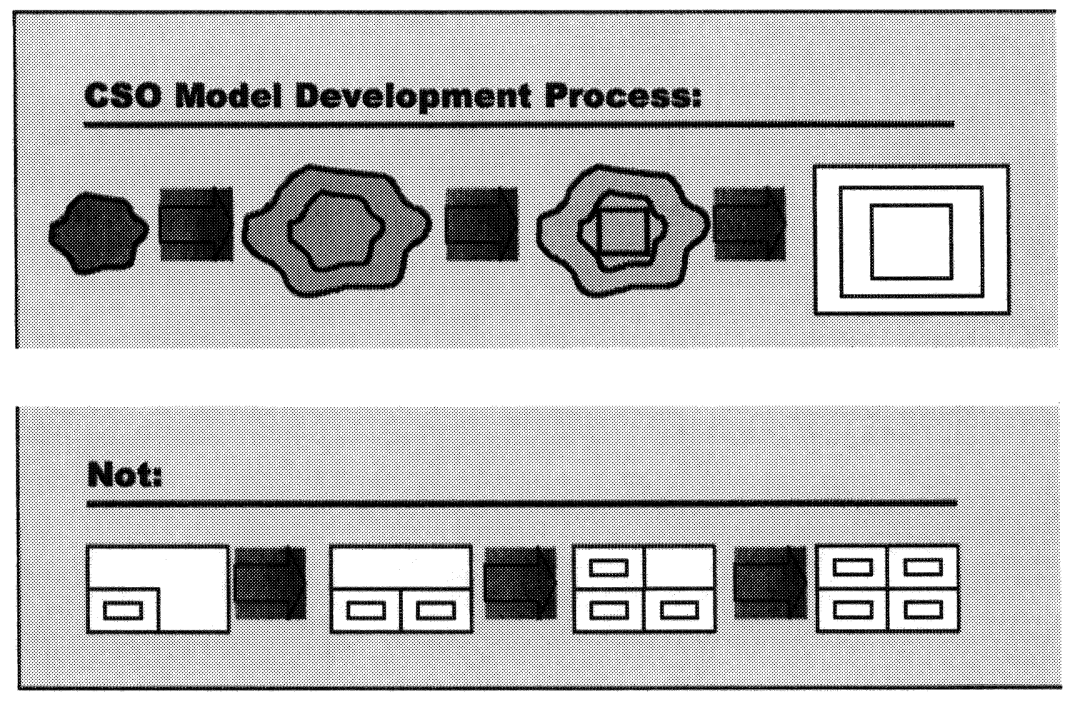

Figure 1.1 Conceptual view of the sewershed model development process.

combined sewer interceptors and separate sewer trunks, including unsteady flow, surcharging, branched and looped pipe networks, pumps, variable orifices, and weirs. The initial characterization of the interceptor and major trunk sewer network is detailed to enable the characterization of system overflows and to support maximization of wet-weather flow capture for treatment. Model representation of the sewershed hydraulic and hydrologic elements is less detailed in the process because less detail is required at this point. However, as the models evolve, the model representation of the sewersheds takes on more and more detail, and as it does so, the modeling system takes on different forms.

The initial sewershed hydrologic representation is in the form of simple ramp-function hydrographs loaded directly to EXTRAN. The ramp functions are used in a systems-analysis approach to understanding interceptor, and eventually, trunk sewer hydraulics. Later in the process, the combined sewersheds are modeled in the United States Army Corps of Engineers (USACOE) Storage, Treatment, Overflow, Runoff Model (STORM), or the updated version of that model, CDM NetSTORM, providing a more detailed characterization of the hydrologic response of the system with a simple algorithm for the computation of rainfall excess. STORM thereby provides a relatively coarse-level wet weather characterization that is useful for initial assessment of impacts and for planning-level alternatives screening used to establish the direction for detailed facility planning and design. Finally, the wet 
weather system model will take on the form of a SWMM model of the entire system that can be used for continuous simulation, using different SWMM blocks for different elements of the system.

There is general agreement in the modeling community that single event or design storm simulations are not sufficient for the generation of long-term CSO statistics, including average annual frequency and volume (EPA, 1993). Continuous simulation more thoroughly accounts for antecedent conditions and inter-event conditions within the system. Continuous simulation is most efficiently accomplished using the single-event EXTRAN model of the interceptor sewer system to set up a continuous simulation form of that model on this portion of the system, and SWMM RUNOFF on the sewersheds. This combination provides more precise (and more spatially detailed) sewer system overflow estimates and more detailed alternatives analysis for development of the long-term plan. The steps in the evolution of the wet weather model are depicted graphically in Figure 1.2.

\subsection{Development of the Tier 1 Modeling System}

For the Tier 1 models, USEPA SWMM-EXTRAN is used in a systemsengineering approach, following a typical systems-engineering construct (Thomann, 1972). In this application, the input is the ramp hydrograph entering the regulating chamber which "accepts" this input and the regulators, interceptor, treatment works and other sewer appurtenances are the system that "operates" on the input and "transforms" the input into output, namely interceptor flow and overflows. The action of the transformation is accomplished by the physical configuration of the interceptor-treatment system, as represented in the EXTRAN hydraulic model. The components of the system are: the ramp input or "forcing function," the output or "response function" and the transformation which is accomplished by the complex interactions among the various system parameters, like pipe shape characteristics, slope, roughness, etc.

The purpose of formulating the problem in terms of the system components is to identify more clearly those phenomena that are "causative," those that are "responsive," and those that act as links or transforming elements between cause and effect. This is the key point in the decision to take this approach. By employing ramping functions for the hydrograph as the exciter, or the forcing function that perturbates the hydraulic system, the complexities of the hydraulic system can be isolated from complexities of sewershed characteristics and their effects on rainfall-runoff hydrographs. Therefore, the 


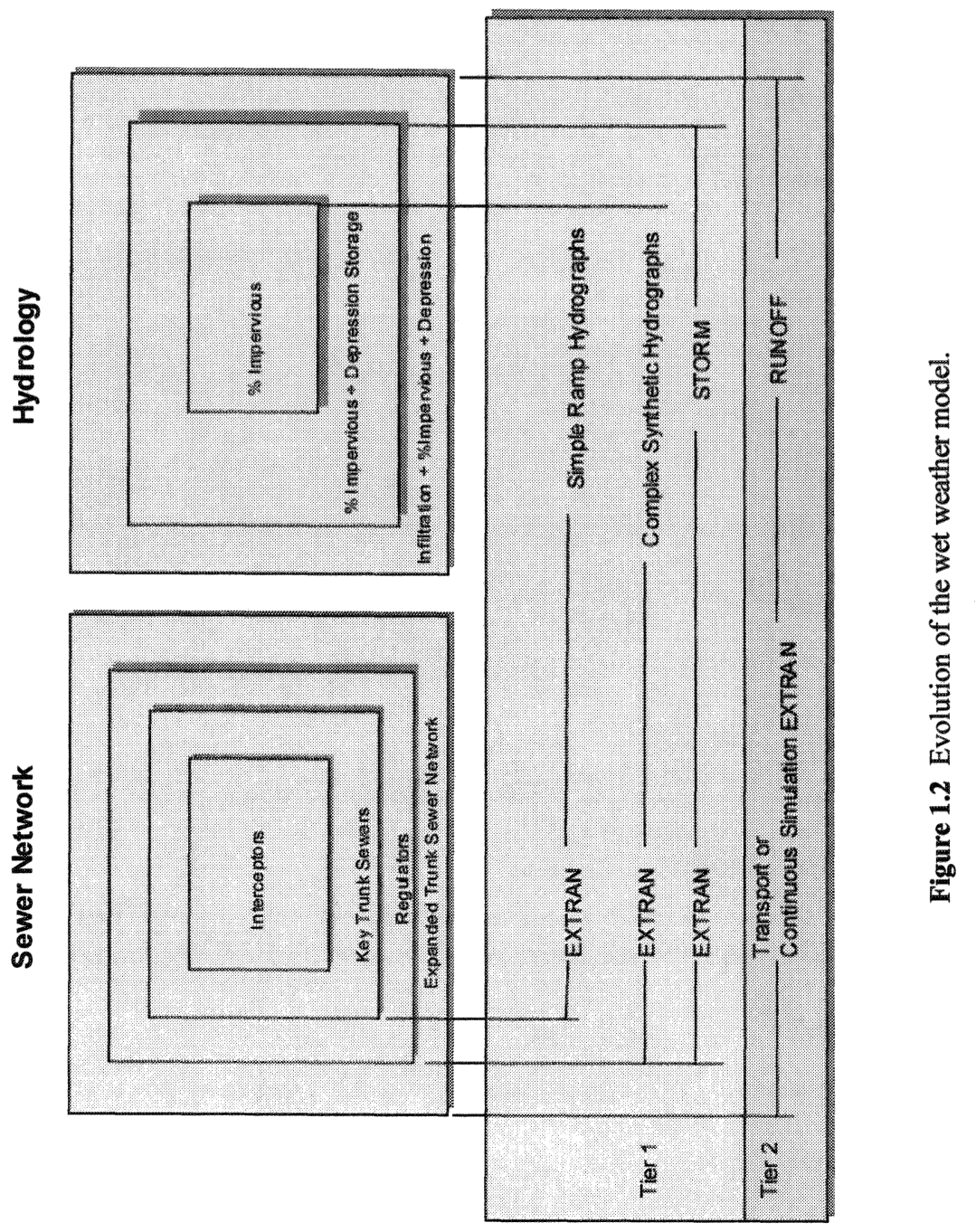


complexities of the interceptor and overflow system hydraulics can be isolated and better elucidated. The complexities of the hydrographs are introduced later in the analysis process through the use of the STORM model in estimating annual overflow volume, frequency and capture statistics for the system.

Rainfall monitoring and sanitary sewer flow monitoring are used to develop rainfall-dependent inflow and infiltration relationships to represent the hydrologic contributions from separate sewered areas. In Tier 1, these relationships were incorporated both into the synthetic hyetograph ramps used in EXTRAN and directly in the NetSTORM model.

The Tier 1 models are used for the hydraulic characterization of interceptors and regulators to a fine level of detail. The model supports estimates of sewer system overflow characteristics using NetSTORM. This characterization of the combined sewersheds and trunk sewer system is at the correct level of detail for the hydrologic and hydraulic characterization requirements of NPDES permits for CSO and sanitary sewer facilities and for the alternatives analyses required for long term CSO control and SSO elimination planning. Often, these characterization reports are required by regulatory agencies as early as the first year of a large and complex project and the Tier 1 model suite typically can be developed in time to meet that need.

\subsection{Development of the Tier 2 Modeling System}

The next phase of the modeling effort, the Tier 2 models, focuses on adding finer detail to various hydraulic and hydrologic elements of the physical system. The intent is to evolve the models to meet the more demanding requirements of facility planning for the design and implementation phases of long term wet weather control planning, and for refined overflow statistics predictions for NPDES permit compliance purposes.

This second phase of the modeling effort is intended to focus on the representation of the various hydrologic abstractions that influence the generation of rainfall excess from the pervious portions of the sewersheds. The most significant of these abstractions is infiltration losses, parameterized for Tier 1 in NetSTORM's modified Rational Method formulation within the lumped "C" coefficient, and represented in Tier 2 as a set of distributed parameters in SWMM RUNOFF using either the Horton or Green-Ampt methods. Linking RUNOFF with EXTRAN in this latter phase enables the initial calibration of EXTRAN performed for dry-weather flows in Tier 1 to be refined using wetweather flows in Tier 2. Initial hydrologic (RUNOFF) calibrations are performed as part of the Tier 2 activities using data available from stage and flow monitors. 
For the Tier 2 models, refinements of the rainfall-dependent inflow and infiltration relationships are used to represent the hydrologic contributions from the separate sewered areas directly, using the RUNOFF $r, t$, and $k$ parameters. These parameters together represent the shape of the surface runoff hydrograph produced by a storm.

The Tier 2 models also provide a more detailed representation of the routing of flows within the sewersheds, enabling in-system storage to be computed directly for the modeled sewers, and the influence of this storage on the routing and overflow of combined wastewater to be simulated more precisely than in STORM. This is accomplished by moving the wet weather model of the sewer system from STORM or NetSTORM to SWMM. Once this effort has been completed, the system is represented entirely within SWMM. RUNOFF block simulations of the hydrologic response, are used to load hydrographs to EXTRAN and to the newer enhanced continuous simulation version of EXTRAN.

The Tier 1 EXTRAN model of the interceptor sewer network developed under Phase 1 is migrated to a newly developed enhanced (parallel-processing with conditional solution-switching) continuous-simulation version of the EXTRAN model for Phase II (Burgess et al., 2000). EXTRAN simulations of single events are used to generate the model representation of the trunk and interceptor system in the enhanced continuous simulation version of EXTRAN, which then are used to perform continuous simulations of the sewer system. This approach is preferable to using the same linked RUNOFF and EXTRAN models in continuous mode, because that requires excessive computer runtimes, often on the order of days or weeks for large systems, and creates output data sets so large that it is impractical to properly "debug" and analyze for valid model representation (e.g. numerical stability, etc.).

The results from detailed hydraulic simulations in EXTRAN can be used effectively in building the enhanced EXTRAN model of the system, constituting a much more efficient continuous simulation model by employing conditional solution switching to restrict full dynamic computations of flow and head when not required (during dry periods when system hydraulics have stabilized). In addition to enabling more efficient simulations of the system, the continuous simulation version of EXTRAN offers other advantages - among which is the capability to support detailed modeling of in-system storage. Storage nodes are defined using detailed stage-volume and stage-discharge relationships. The EXTRAN model is used to provide accurate modeling of the interceptor/trunk sewer hydraulics based on detailed single-event (synthetic ramped hyetograph) simulations for spatially uniform runoff inflow rates (on a unit area basis), the results of which are integrated into the continuous-simulation EXTRAN model for continuous simulations. 
The precision of the input data for a number of specific parameters also is refined in the Tier 2 models. In these cases, the model representation (i.e. the manner in which the parameter is simulated) may not change, but specific values used in the model may change, to reflect the availability of more detailed information. For example, parameters refined in the Tier 2 models include the sewershed-specific wastewater flow rates and the sewershed-specific impervious cover allocations. These refinements result from ongoing review and refinement of the land-use and water consumption databases. Detailed quality assurance review of this large and complex hydraulic model is an ongoing process that will result in modifications and refinements to the model, and will continue to be an emphasis of the model development process.

\subsection{Use of Monitoring Data}

The linked RUNOFF-continuous simulation EXTRAN model of the combined sewer system may be applied with the same long-term airport rainfall records (usually available for approximately 50 years of hourly simulation in most places) used for STORM/NetSTORM applications during Tier 1. In the Tier 2 context, these input data can be used in the model to generate sewer system overflow characteristics and to support long-term planning. In addition to the long-term hourly record, data collected at 5 to 15 -minute intervals at local rain gauges allows Tier 2 models to be used in simulations for whatever period of record is available. For the Tier 2 models, it is more common in recent years to make use of recorded rainfall data that are calibrated for larger storms using NEXRAD radar data. Application of uniform or averaged rainfall intensities over the land surface area is sometimes satisfactory for long-term simulations but tends to eliminate precipitation fluctuations that are reflected in the system's hydrologic response. A NEXRAD modified rainfall record allows a greater degree of precision and spatial detail in hydrologic modeling, and is particularly effective in improving the model calibration and verification process. Greater temporal and spatial detail allows more accurate calibration of sewershed hydrologic and interceptor hydraulic conditions during and after individual storms. The use of this spatially synoptic rainfall information is consistent with the philosophy of expanding the complexity of the modeling system as it evolves from Tier 1 to Tier 2 .

Typically for Tier 2 modeling calibration efforts, multiple years of rainfall, interceptor hydraulic grade, interceptor flow, flow at combined sewer locations, separate trunk sewer flow for inflow and infiltration analyses, and endof-trunk sewer flow monitoring are used. Portable flow meters deployed and rotated continuously during an extended period of several or more years at 
numerous sewer locations throughout the service area allows investigators to not only elucidate regional base wastewater flow and rainfall-runoff characteristics, but also to characterize inter-seasonal and inter-annual patterns of groundwater infiltration to the sewer system. If the project allows this longterm commitment to sewage flow characterization, it is superior to situations where a short-period, intensive spatial and temporal synoptic monitoring is mandated by project constraints. It is much more difficult to build models that adequately can represent the important inter-seasonal and inter-annual groundwater infiltration variability without long-term monitoring information.

Continued rainfall monitoring and sanitary sewer flow monitoring allow for the further development and refinement of the rainfall-dependent inflow and infiltration relationships that are critical to the proper representation of the hydrologic contributions from the separate sewered areas in Tier 2 models.

\subsection{Uses of the Modeling System}

Tier 1 models provide a basis for initial characterization of the hydrology and hydraulics of the sewage interceptor transmission and treatment system. They also provide system-wide estimates of the frequency, duration, and volume of CSOs adequate for NPDES Phase I permit compliance. Sewerage agencies also can make use of the Tier 1 models in responding to technical questions regarding the operating capacity of interceptors, trunks and treatment systems and the capability of that infrastructure to provide future service needs. Tier 1 models are used extensively in conceptual planning for system expansions such as proposed relief sewers. The Tier 1 modeling system is used to support alternatives selection for regional long term wet weather control concept planning both regionally, basin-wide, and in individual planning basins. They also have proved useful in innovative programs to provide quarterly CSO discharge estimates for NPDES permit reporting in lieu of expensive overflow monitoring programs.

Tier 2 models allow the refining and updating of the hydrologic and hydraulic characterization of the regional sewer system, including refining and updating system-wide estimates of the frequency, duration, and volume of CSO's. They are used to assist sewerage agencies in responding to technical questions regarding the operating capacity of interceptors and trunk sewers, and in defining wet weather transport conditions affecting the operation of the sewage treatment plant and the capability to provide future service needs. The Tier 2 models are used to support facility planning and design phases for the implementation wet weather overflow control planning, including CSO and sanitary sewer enhancement programs. A truly innovative use for the Tier 2 
model system is to provide refined quarterly CSO discharge estimates for NPDES permit reporting in lieu of monthly overflow monitoring and reporting. This idea is gaining acceptance within the regulatory community as they attempt to respond to the needs of the regulated agencies to minimize the impact of compliance-related costs on the customer base.

\section{References}

Burgess, E., W.R. Magro, M. Clement, C. Moore and J. Smullen. 2000. "Parallel Processing Enhancement to SWMM/EXTRAN." Journal of Water Management Modeling R206-03. doi: 10.14796/JWMM.R206-03.

EPA. 1993. Manual: Combined Sewer Overflow Control,EPA/625/R-93/007,USEPA, Washington, D.C.

Roesner, L.A., J.A. Aldrich and R.E. Dickinson. 1988. Storm Water Management Model User's Manual, Version 4: Addendum I. EXTRAN; Cooperative Agreement CR-811607, U.S. EPA, Cincinnati, Ohio.

Thomann, R.V. 1972. Systems Analysis and Water Quality Management. McGrawHill Book Company, New York, NY. 285 pp. 